\title{
Acute unilateral reexpansion pulmonary edema after pleuropulmonary decortication
}

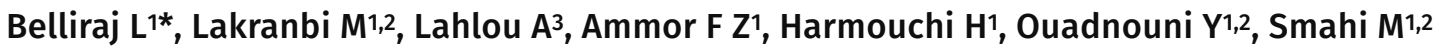

\author{
* Correspondence \\ Belliraj Layla \\ Department of thoracic surgery \\ University hospital Hassan II Fes \\ Morocco \\ Email: lbelliraj@yahoo.fr \\ 1Department of Thoracic Surgery, \\ University Hospital Hassan II, Fes, \\ Morocco. \\ 2Faculty of Medicine and Pharmacy, \\ University Sidi Mohamed Ben Abdellah, \\ Fes, Morocco. \\ ${ }^{3}$ Departement of Intensive Care A4, \\ University Hospital Hassan II, Fes, \\ Morocco. \\ Received: Jan 19, 2019 \\ Accepted: Apr 24, 2019 \\ Published: May 7, 2019
}

\begin{abstract}
The development of unilateral pulmonary edema at the time of reexpansion is a rare complication often associated with aspirational drainage of a pneumothorax. It has been described exceptionally in the postoperative course of a pleural surgery. The main involved factors are prolonged atelectasis, reexpansion pulmonary effusion, the importance and duration of pleural effusion.

This complication must be known to anesthesiologist during thoracic surgery for its mortality, which is evaluated at 20\% in the litterature and that an early diagnosis allows the an effective treatment.

We report an acute respiratory distress in the immediate postoperative course of pleuropulmonary decortication for a recurrent tuberculous pleurisy.
\end{abstract}

Keywords: Unilateral Pulmonary Edema, Pneumothorax, Pleural Effusion.

The search for Koch's bacillus in the sputum was negative. Face to this clinical situation, surgery was decided for diagnosis and treatment.

The pre-anesthetic examination found a patient in good general condition, polypneic at 19 cycles / minute, his $\mathrm{SpO} 2$ at $92 \%$ in ambient air, a normal blood pressure, and a heart rate at 95 beats / minute. Thorax auscultation revealed a fluid pleural effusion syndrome throughout the right hemithorax and the left basithoracic region.

The radiological assessment showed an opaque right hemithorax and a left pleural effusion with no mediastinal discharge (Figure 1).

The preoperative preparation consisted of incitative spirometry and evacuation puncture of the right pleural effusion, which brought about $500 \mathrm{ml}$ of yellow fluid with the same radiological images on the control chest X-ray.

exudative fluid and the pleural biopsy was inconclusive. 


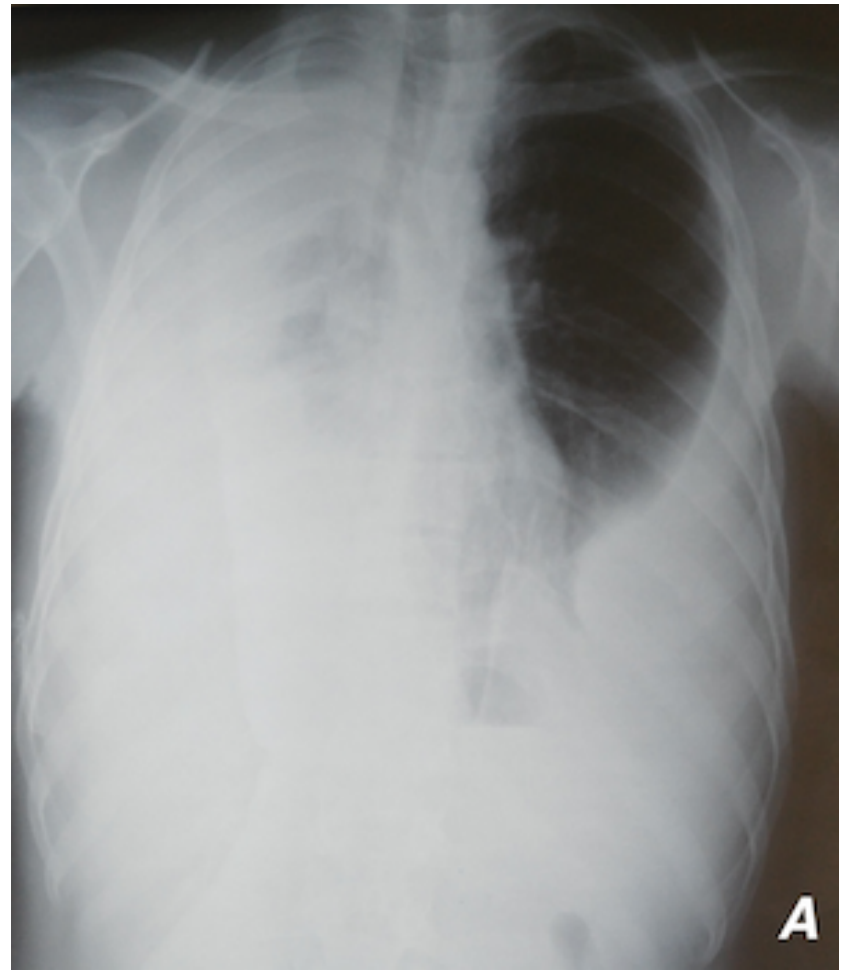

Figure 1.A: Chest X-ray showing a right opaque hemithorax with a left pleurisy.

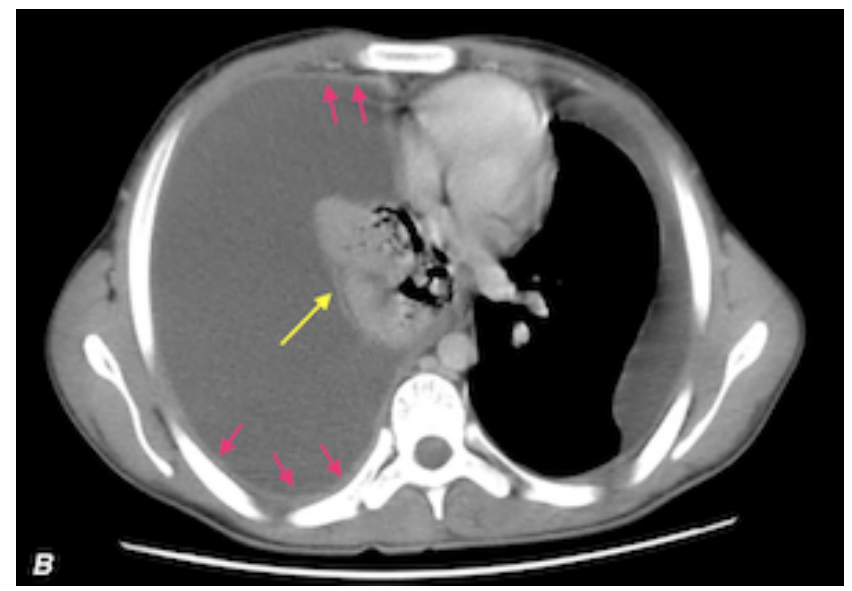

Figure 1.B: Thoracic CTscan showing bilateral pleurisy with right pachypleuritis (red arrow) and right lung atelectasis (yellow arrow).

The patient was admitted to the operating room, with standard monitoring including a 95\% saturation in the ambient air. The procedure lasted 2 hours. It consisted in a pleuro-pulmonary decortication after aspiration of $1000 \mathrm{ml}$ of yellow liquid. The exploration found a thickened pleura dotted with white pleural nodules.

At the end of the surgery, there was a desaturation at $75 \%$ and a tachypnea with aspiration of foamy and pink secretions via the tracheal tube. The chest auscultation revealed the presence of crackles, and arterial blood gas (ABG) showed a $\mathrm{PaO} 2$ / FiO2 ratio at 270 .

An urgent chest $X$-ray showed multiple nodular alveolar opacities, poorly limited and diffuse, throughout the right lung and therefore the diagnosis of RPE was confirmed (figure2).

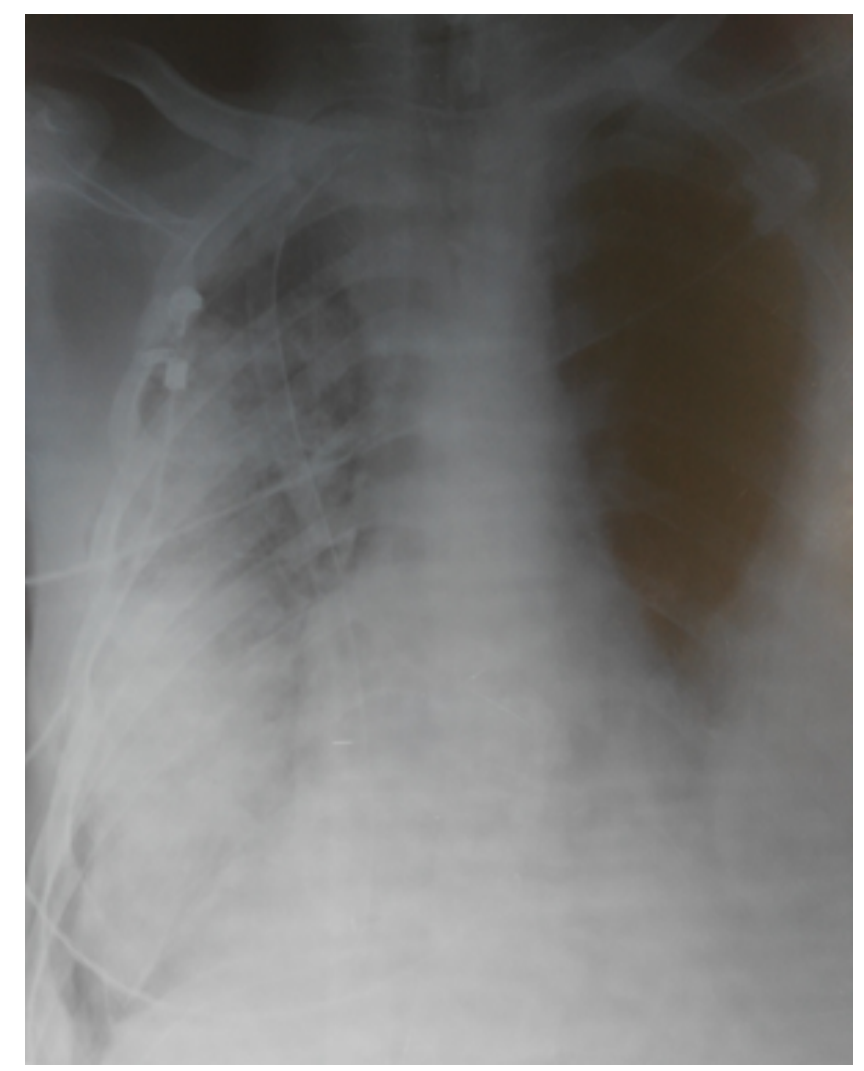

Figure 2: Chest X-ray showing right alveolar syndrome.

The patient was put on diuretics with increase in positive expiratory pressure allowing his extubation in post-interventional surveillance room.

After a stay in intensive care unit for $48 \mathrm{~h}$, with close noninvasive ventilation sessions, a respiratory physiotherapy and diuretics, the evolution was progressively favorable with a decrease of the crackles, an improvement of the gasometric parameters and a regression of the radiological images (figure3) [2].

The histopathological study revealed an aspect of pleural tuberculosis and the patient was put on antibacillary treatment with a good clinical and radiological evolution. 


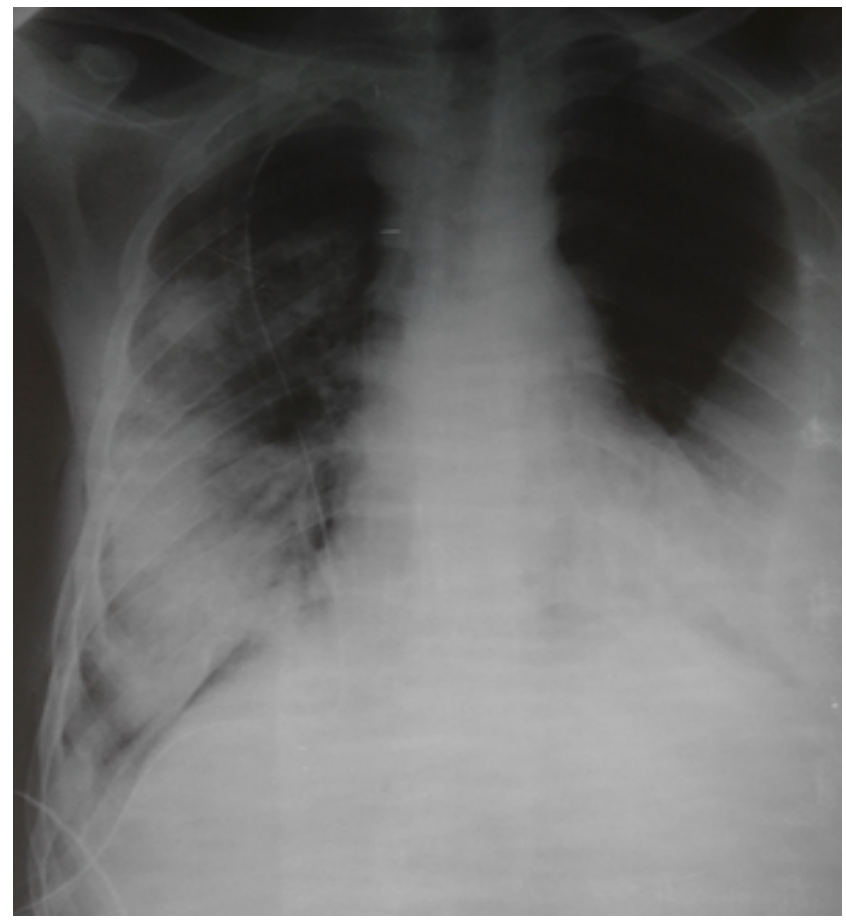

Figure 3: Chest $\mathrm{X}$-ray showing regression of the lesions.

\section{Discussion}

Acute RPE, also known as "vacuo" pulmonary edema, is a rare complication, typically described after thoracic drainage. However, its installation immediately after pleuropulmonary decortication is an exceptional situation, reported only once by Lemoine et al [3]]. Whatever its etiology, the diagnosis must be made early, based on a set of clinical and radiological data. In the case of our patient the clinical condition was typical of acute pulmonary edema in its severe form of respiratory distress, desaturation, foamy sputum, crackles on lung auscultation, a very evocative radiology image and a $\mathrm{PaO} 2$ / FiO2 ratio <300 on $\mathrm{ABG}$ that appeared at the end of the intervention.

Several pathophysiological mechanisms explain this acute RPE. In our patient the main mechanisms involved were the long duration of pulmonary collapse, the volume of pleural fluid, and immediate pulmonary reexpansion.

Therapeutic management relies essentially on adequate oxygenation and redistribution of liquids to capillaries and interstitial spaces through an increase in intra-alveolar pressure. Diuretic-based medical treatment remains the treatment of choice in emergency situation to fight against the signs of respiratory distress associated with certain urgent measures such as adapted oxygen therapy and the half-sitting position. In the case of thoracic drainage, the pleural suction should be stoped immediately [4].

The recommended prevention (fractional evacuation without depression and with slow reexpansion) is difficult in thoracic surgery [3].

The prognosis will depend on the speed of diagnosis and the quality of care. The mortality varies from 5 to $20 \%$ depending on the series $[\underline{4}, \underline{5}]$.

\section{Conclusion}

Acute RPE is a serious complication that must be known by all anesthesiologists during thoracic surgery. The case presented here underlines the interest of the speed of the diagnosis and management, allowing a fast and favorable evolution.

\section{Abbreviations}

- Arterial blood gas :ABG

- Fraction of inspired oxygen :FiO2

- Peripheral capillary oxygen saturation :SpO2

- Partial pressure of oxygen : PaO2

- Reexpansion pulmonary edema :RPE
}

\section{Conflict of interests}

The authors declare no conflict of interest.

\section{Acknowledgments}

We thank the Intensive Care team A4 of the University Hospital HASSAN II of Fes.

\section{Copyrights}

(c) Belliraj Layla et al, 2019; licensee OA Journal of Clinical Case Reports. This is an Open Access article distributed under the terms of the Creative Commons Attribution License (http:// creativecommons.org/licenses/by/4.0), which permits unrestricted use, distribution, and reproduction in any medium, provided the original work is properly credited.

\section{References}

1. Rabiou S, Lakranbi M, Ghalimi J, et al. (2015) OEdème aigu pulmonaire unilobaire supérieur gauche après drainag thoracique. Ann Fr Méd D'urgence. 5:265-266.

2. Angel G, Andreu JM, Aulagnierl V, et al. (1997) E-dème pulmonaire de réexpansion après exérèse d'une tumeur intrathoracique. Ann Fr Anesth Reanim. 16:370-373.

3. Lemoine E, Martin L, Robert M, et al. (1992) Edème pulmonaire unilatéral de réexpansion postopératoire. Ann $\mathrm{Fr}$ Anesth Reanim. 11:464-466.

4. Shimi A, Madani H, Fatima ZB, et al. (2009) OEdème pulmonaire unilatéral de réexpansion après drainage thoracoscopique d'une pleurésie récidivante. Web-Anesthésie. Réanimation. 4: $1-4$.

5. Mahfood S, HIX W R, Aaron B L, et al. (1988) Re-expansion pulmonary edema. Ann Thorac Surg. 4: 340-345. 